\title{
On the gain of collaboration in a two dimensional ruin problem
}

\section{Peter Grandits ${ }^{1}$}

Received: 27 July 2018 / Revised: 10 January 2019 / Accepted: 24 January 2019 /

Published online: 12 February 2019

(c) The Author(s) 2019

\begin{abstract}
We investigate the following problem: the endowment process of two insurance companies is given by a two dimensional Brownian motion with drift. How big is the gain in the probability that both companies survive, if they collaborate optimally by transfer payments, in comparison to the non collaboration case. We provide an explicit formula for the value function of the problem.
\end{abstract}

Keywords Ruin probabilities · Optimal control problem · Collaboration · Twodimensional Brownian motion

Mathematics Subject Classification Primary 49J20 · 35R35 · Secondary 91B70

\section{Introduction}

Whereas the literature on the two main evaluation criteria for insurance companies, namely the ruin probabilities and the maximal possible dividend payments, is vast in the one dimensional case, i.e., if one considers only one company, see e.g. for an overview $[1,3]$, there are not so many results in the two- or multidimensional case. We mention for the dividend problem, [2, 6-8], where it is investigated, how the total dividend payments of two companies can be optimized, if they collaborate.

We want to investigate a similar problem for the ruin probabilities in this paper. Assuming that both companies can collaborate by transfer payments, and assuming that it is their goal that both companies survive, we want to examine how big the gain of collaboration in terms of this surviving probabilities is in comparison to the non-collaboration case.

Peter Grandits

pgrand@fam.tuwien.ac.at

1 Institut für Stochastik und Wirtschaftsmathematik, TU Wien, Wiedner Hauptstraße 8-10, 1040 Vienna, Austria 
The endowment processes will be modeled by two dimensional Brownian motion with drift, and we assume that the transfer process is absolutely continuous with respect to the Lebesgue measure.

It will turn out that the problem is a stochastic control problem, and let us mention that an equivalent problem is considered in [9], where the authors viewed the problem as tax policy problem. We will show the equivalence in the next section, where we introduce our model. McKean and Shepp mention in their paper (page 6591) that it is known, what the optimal strategy is, but that the value function is unknown. We shall provide an explicit formula for this value function.

Let us finally cite the papers $[4,5]$, where one can also find some results on multidimensional ruin problems.

\section{The model}

We describe the endowment of the two companies by the stochastic processes $X_{t}$ and $Y_{t}$, which fulfill

$$
\begin{aligned}
& d X_{t}=\mu_{1} d t+d W_{t}^{(1)}+c_{t} d t, \\
& d Y_{t}=\mu_{2} d t+d W_{t}^{(2)}-c_{t} d t,
\end{aligned}
$$

with initial conditions $X_{0}=x$ and $Y_{0}=y$, and independent standard Brownian motions $W_{t}^{(1)}, W_{t}^{(2)}$. We assume positive drift rates, i.e. $\mu_{1}, \mu_{2}>0$. The drift rate $c_{t}$ has the interpretation of a transfer process from one company to the other, which means that for positive $c_{t}$ we have a transfer from the second company to the first one, and for negative $c_{t}$ it is vice versa. Furthermore, we assume that-for safety reason-each company keeps a positive drift rate of at least $\delta>0$, which should satisfy $\delta<\min \left(\mu_{1}, \mu_{2}\right)$, i.e. we require $\mu_{1}+c_{t} \geq \delta$ and $\mu_{2}-c_{t} \geq \delta$ or

$$
c_{t} \in\left[-\mu_{1}+\delta, \mu_{2}-\delta\right] \text {. }
$$

The target functional is given by the probability that both companies survive, i.e.

$$
J(x, y, c)=\mathbf{P}\left(\tau_{1}=\infty, \tau_{2}=\infty\right),
$$

where $\tau_{1}$ and $\tau_{2}$ denote the ruin probabilities for $X_{t}$, resp. $Y_{t}$, i.e.

$$
\begin{aligned}
& \tau_{1}:=\inf \left\{t>0 \mid X_{t}=0\right\}, \\
& \tau_{2}:=\inf \left\{t>0 \mid Y_{t}=0\right\} .
\end{aligned}
$$

Finally, we introduce our value function

$$
V(x, y)=\sup _{c \in \mathcal{A}} J(x, y, c),
$$

where $\mathcal{A}$ denotes the admissible strategies, which we take as the progressively measurable processes with respect to the filtration generated by our two dimensional Brownian motion, and fulfilling (2). 
We now introduce the new control variable $u_{t}:=\mu_{1}+c_{t}$, implying-with $\bar{\mu}:=\mu_{1}+\mu_{2}-$

$$
\begin{aligned}
& d X_{t}=u_{t} d t+d W_{t}^{(1)}, \\
& d Y_{t}=\left(\bar{\mu}-u_{t}\right) d t+d W_{t}^{(2)},
\end{aligned}
$$

with $u_{t} \in[\delta, \bar{\mu}-\delta]$, which is, for $\bar{\mu}=1$, the same model as in the paper by McKean and Shepp [9], which we have mentioned in the introduction.

\section{Construction of a classical solution for the Hamilton-Jacobi- Bellman equation}

For the Hamilton-Jacobi-Bellman (HJB) equation and the boundary conditions in our extremal problem we find

$$
\begin{aligned}
& \max _{u \in[\delta, \bar{\mu}-\delta]}\left\{u V_{x}+(\bar{\mu}-u) V_{y}+\frac{1}{2} \Delta V\right\}=0, \\
& V(x, 0)=V(0, y)=0, \\
& V(x, \infty)=1-e^{-2(\bar{\mu}-\delta) x}, \\
& V(\infty, y)=1-e^{-2(\bar{\mu}-\delta) y},
\end{aligned}
$$

where the PDE in (7) holds in $G:=\{(x, y) \mid x>0, y>0\}$. We shall construct an explicit $C^{2}$-solution for (7), which is symmetric with respect to the first median. We start with the solution of

$$
\begin{aligned}
& \max _{u \in[\delta, \bar{\mu}-\delta]}\left\{u V_{x}+(\bar{\mu}-u) V_{y}+\frac{1}{2} \Delta V\right\}=0, \\
& V(0, y)=0, \\
& V(x, \infty)=1-e^{-2(\bar{\mu}-\delta) x}, \\
& V_{x}(t, t)=V_{y}(t, t), t>0,
\end{aligned}
$$

where the PDE here holds in $H:=\{(x, y) \mid x>0, y>x\}$, i.e in the upper half of $G$. Once we have found this solution, we mirror it on the first median and show that this gives a full $C^{2}$-solution, especially $C^{2}$ at the first median.

We start to construct a solution of

$$
\begin{aligned}
& (\bar{\mu}-\delta) V_{x}+\delta V_{y}+\frac{1}{2} \Delta V=0, \\
& V(0, y)=0 \\
& V(x, \infty)=1-e^{-2(\bar{\mu}-\delta) x}, \\
& V_{x}(t, t)=V_{y}(t, t), t>0,
\end{aligned}
$$


on $H$, and show then that we have $V_{x} \geq V_{y}>0$ on $H$, which implies that the solution of (9) is also a solution of (8). The interpretation of this is that one should give all the drift to the weaker company, apart from the reserve $\delta$.

Let us start with the ansatz

$$
V(x, y)=\hat{V}(x)+\tilde{V}(x, y),
$$

with $\hat{V}(x)=1-e^{-2(\bar{\mu}-\delta) x}$, which gives the following problem for $\tilde{V}$.

$$
\begin{aligned}
& (\bar{\mu}-\delta) \tilde{V}_{x}+\delta \tilde{V}_{y}+\frac{1}{2} \Delta \tilde{V}=0, \\
& \tilde{V}(0, y)=0, \\
& \tilde{V}(x, \infty)=0, \\
& \left(\tilde{V}_{x}-\tilde{V}_{y}\right)(t, t)=\left(\hat{V}_{y}-\hat{V}_{x}\right)(t, t), t>0 .
\end{aligned}
$$

We have the following Proposition.

Proposition 3.1 The function $\tilde{V}(x, y)=\frac{\delta-\bar{\mu}}{\delta}\left(e^{-(\bar{\mu}-2 \delta) x}-e^{-\bar{\mu} x}\right) e^{-\bar{\mu} y}$ solves the system (11).

Before we prove the proposition, let us remark that we could, of course, verify Proposition 3.1 by just plugging in the explicit solution formula. But we want to demonstrate, how we found the solution.

Proof To solve the system (11), we try the separation ansatz $\tilde{V}(x, y)=f(x) g(y)$. Plugging into the first equation of (11), provides, after some rearrangements, the following two equations

$$
\begin{aligned}
& 2(\bar{\mu}-\delta) \frac{f^{\prime}(x)}{f(x)}+\frac{f^{\prime \prime}(x)}{f(x)}=\lambda, \\
& -2 \delta \frac{g^{\prime}(y)}{g(y)}-\frac{g^{\prime \prime}(y)}{g(y)}=\lambda .
\end{aligned}
$$

We assume now that

$$
\lambda \neq \delta^{2},
$$

which we shall verify later on, by choosing $\lambda$ in an appropriate way. For the second of these equations we get the solution

$$
g(y)=C_{1} e^{\theta_{1} y}+C_{2} e^{\theta_{2} y},
$$

with real constants $C_{1}, C_{2}$ and the roots $\theta_{1,2}=-\delta \pm \sqrt{\delta^{2}-\lambda}$, where the positive sign corresponds to $\theta_{1}$. 
Similarly, using the boundary condition $f(0)=0$, stemming from the second equation of (11), we get as solution for the first equation in (12)

$$
f(x)=C_{3}\left(e^{\gamma_{1} x}-e^{\gamma_{2} x}\right),
$$

where $C_{3}$ is a real constant, and $\gamma_{1,2}=-(\bar{\mu}-\delta) \pm \sqrt{(\bar{\mu}-\delta)^{2}+\lambda}$ holds. Again, we shall verify later on that

$$
(\bar{\mu}-\delta)^{2}+\lambda \neq 0
$$

is true, for the $\lambda$ we shall choose, giving two distinctive roots.

For the fourth equation of (11) we get

$$
\left(\tilde{V}_{x}-\tilde{V}_{y}\right)(t, t)=-2(\bar{\mu}-\delta) e^{-2(\bar{\mu}-\delta) t} .
$$

Plugging (14) and (15) into this equation we arrive at

$$
\begin{aligned}
& C_{1} C_{3}\left(\gamma_{1}-\theta_{1}\right) e^{\left(\gamma_{1}+\theta_{1}\right) t}+C_{2} C_{3}\left(\gamma_{1}-\theta_{2}\right) e^{\left(\gamma_{1}+\theta_{2}\right) t}+ \\
& C_{1} C_{3}\left(-\gamma_{2}+\theta_{1}\right) e^{\left(\gamma_{2}+\theta_{1}\right) t}+C_{2} C_{3}\left(-\gamma_{2}+\theta_{2}\right) e^{\left(\gamma_{2}+\theta_{2}\right) t}=-2(\bar{\mu}-\delta) e^{-2(\bar{\mu}-\delta) t} .
\end{aligned}
$$

We now solve (18) more or less by trial and error, i.e. we set $C_{1}=0, \theta_{2}=\gamma_{2}$, which gives as necessary condition

$$
C_{2} C_{3}\left(\gamma_{1}-\gamma_{2}\right) e^{\left(\gamma_{1}+\gamma_{2}\right) t}=-2(\bar{\mu}-\delta) e^{-2(\bar{\mu}-\delta) t} .
$$

The exponential function of the r.h.s. and the 1.h.s. coincide, because of the explicit known values of $\gamma_{1}$ and $\gamma_{2}$. The condition $\theta_{2}=\gamma_{2}$ gives, after some lengthy but completely elementary calculation, the equation

$$
4 \lambda^{2}+4 \lambda \bar{\mu}^{2}-8 \lambda \bar{\mu} \delta=0
$$

and we choose as solution

$$
\lambda=-\left(\bar{\mu}^{2}-2 \bar{\mu} \delta\right)<0,
$$

where the inequality holds, because of our assumption $\delta<\min \left(\mu_{1}, \mu_{2}\right)$. This choice guarantees that (13) and (16) are true, and gives the following values for the characteristic roots

$$
\begin{aligned}
& \theta_{2}=\gamma_{2}=-\bar{\mu}, \\
& \theta_{1}=-\gamma_{1}=\bar{\mu}-2 \delta .
\end{aligned}
$$

Using these values, we see that $C_{2} C_{3}=\frac{\delta-\bar{\mu}}{\delta}$ is sufficient for the validity of (19). We choose $C_{3}=1$ and $C_{2}=\frac{\delta-\bar{\mu}}{\delta}$. Hence, (19) is now true, therefore the fourth equation 
of (11) is valid. This implies that until now the first, the second and the fourth equation of (19) are satisfied by our construction, and we remain with the third one.

Here we have to check that $g(\infty)=0$ is true. But this holds, because of $C_{1}=0, \theta_{2}=-\bar{\mu}<0$. Finally, employing all the explicit values of the constants $C_{i}$ and the characteristic roots, gives the asserted form of the function $\tilde{V}(x, y)$.

Because of (10) an immediate corollary is

\section{Corollary 3.1 The function}

$$
V(x, y)=\left(1-e^{-2(\bar{\mu}-\delta) x}\right)+\frac{\delta-\bar{\mu}}{\delta}\left(e^{-(\bar{\mu}-2 \delta) x-\bar{\mu} y}-e^{-\bar{\mu} x-\bar{\mu} y}\right)
$$

solves (9).

As announced earlier, we now show that the candidate solution of the previous corollary fulfills the HJB system on the set $H$.

Lemma 3.1 The function $V(x, y)$ defined in Corollary 3.1 solves the system (8) on the set $H$.

Proof All we have to show is that $V_{x}(x, y) \geq V_{y}(x, y)>0$ on the set $H$.

Straightforward calculation gives

$$
V_{x}(x, y)-V_{y}(x, y)=2(\bar{\mu}-\delta) e^{-2(\bar{\mu}-\delta) x}\left(1-e^{-\bar{\mu}(y-x)}\right),
$$

which is clearly non negative on the set $H$. To check $V_{y}(x, y)>0$ is also straightforward.

Our next step is to define the candidate function on the rest of the set $G$, i.e. below the first median. We do this by just mirroring at the first median, i.e. we define

$$
V(x, y)=\left(1-e^{-2(\bar{\mu}-\delta) \min (x, y)}\right)+\frac{\delta-\bar{\mu}}{\delta}\left(e^{-(\bar{\mu}-2 \delta) \min (x, y)-\bar{\mu} \max (x, y)}-e^{-\bar{\mu} x-\bar{\mu} y}\right),
$$

and assert

Proposition 3.2 The function defined in (23) solves the system (7) in the set $G$, and is $C^{2}$ there.

Proof All we have to show is that our function is $C^{2}$ at the first median. We denote limits from above the first median by $V((t, t)+)$ and from below by $V((t, t)-)$. A somewhat lengthy but elementary computation provides (continuity is clear)

$$
V_{x}((t, t)-)=V_{x}((t, t)+)=\frac{\bar{\mu}^{2}-\bar{\mu} \delta}{\delta} e^{-2(\bar{\mu}-\delta) t}+\frac{-\bar{\mu}^{2}+\bar{\mu} \delta}{\delta} e^{-2 \bar{\mu} t}
$$


$V_{y}((t, t)-)=V_{y}((t, t)+)$ follows by symmetry. Moreover, we have

$$
V_{x x}((t, t)-)=V_{x x}((t, t)+)=\frac{-\bar{\mu}^{3}+\bar{\mu}^{2} \delta}{\delta} e^{-2(\bar{\mu}-\delta) t}+\frac{\bar{\mu}^{3}-\bar{\mu}^{2} \delta}{\delta} e^{-2 \bar{\mu} t} .
$$

$V_{y y}((t, t)-)=V_{y y}((t, t)+)$ follows again by symmetry, and the same is true for $V_{x y}((t, t)-)=V_{x y}((t, t)+)$.

\section{Verification}

In this section we verify that the function $V(x, y)$ of $(23)$ is indeed the value function of our problem. We have

Theorem 4.1 The function $V(x, y)$ defined in (23) is the value function of the problem (5), and the optimal strategy is given by $u^{*}\left(X_{t}, Y_{t}\right)=(\bar{\mu}-\delta) \mathbf{1}_{\left\{Y_{t} \geq X_{t}\right\}}+\delta \mathbf{1}_{\left\{Y_{t}<X_{t}\right\}}$ (which correspond to $c^{*}\left(X_{t}, Y_{t}\right)=\left(\mu_{2}-\delta\right) \mathbf{1}_{\left\{Y_{t} \geq X_{t}\right\}}+\left(-\mu_{1}+\delta\right) \mathbf{1}_{\left\{Y_{t}<X_{t}\right\}}$ in the original control variable).

Proof Let $u_{t}$ be an arbitrary admissible strategy with values in $[\delta, \bar{\mu}-\delta]$. We consider the process $V\left(X_{t \wedge \tau}, Y_{t \wedge \tau}\right)$, where $\tau:=\tau_{1} \wedge \tau_{2}$, the first time when one of the two companies is ruined.

Ito's formula implies

$$
\begin{aligned}
d V\left(X_{t}, Y_{t}\right)= & V_{x}\left(X_{t}, Y_{t}\right)\left(u_{t} d t+d W_{t}^{(1)}\right)+V_{y}\left(X_{t}, Y_{t}\right)\left(\left(\bar{\mu}-u_{t}\right) d t+d W_{t}^{(2)}\right)+\frac{1}{2} \Delta V\left(X_{t}, Y_{t}\right) d t \\
= & \left(u_{t}\left(V_{x}\left(X_{t}, Y_{t}\right)-V_{y}\left(X_{t}, Y_{t}\right)\right)+\bar{\mu} V_{y}\left(X_{t}, Y_{t}\right)+\frac{1}{2} \Delta V\left(X_{t}, Y_{t}\right)\right) d t \\
& +V_{x}\left(X_{t}, Y_{t}\right) d W_{t}^{(1)}+V_{y}\left(X_{t}, Y_{t}\right) d W_{t}^{(2)} .
\end{aligned}
$$

Now, the drift part is nonpositive because of the validity of our HJB equation in (7), and therefore $V\left(X_{t}, Y_{t}\right)$ as well as $V\left(X_{t \wedge \tau}, Y_{t \wedge \tau}\right)$ are local supermartingales. Because $V$ is bounded, we have in fact a uniformly integrable supermartingale $V\left(X_{t \wedge \tau}, Y_{t \wedge \tau}\right)$. Hence, the supermartingale convergence theorem implies that the limit

$$
\lim _{t \rightarrow \infty} V\left(X_{t \wedge \tau}, Y_{t \wedge \tau}\right)
$$

exists a.s. Moreover, this limit, say $\rho$, clearly has to lie in the interval $[0,1]$. Now, the niveau lines for $\rho \in(0,1)$ are piecewise $C^{1}$ curves and can not - because of the oscillation of the Brownian motions - be limit curves. Hence, the only remaining possibilities are $\rho=0$ and $\rho=1$, where the first corresponds to the case when one of the companies gets ruined, i.e. the set $\{\rho=0\}=\{\tau<\infty\}$, and the other when both become "infinitely rich", i.e. $\{\rho=1\}=\left\{X_{\infty}=\infty, Y_{\infty}=\infty\right\}$. Therefore, one finds 


$$
V(x, y) \geq \mathbf{E}\left[V\left(X_{\tau}, Y_{\tau}\right)\right]=\mathbf{E}\left[V\left(X_{\tau}, Y_{\tau}\right) \mathbf{1}_{\{\tau<\infty\}}+V(\infty, \infty) \mathbf{1}_{\{\rho=1\}}\right]=\mathbf{P}(\tau=\infty),
$$

where the first inequality holds because of the uniformly supermartingale property, and the last equality because of the second,third and fourth equality in (7).

If one uses the optimal strategy $u_{t}^{*}$ instead of the arbitrary admissible $u_{t}$ of the first part of the proof, one gets a martingale instead of a supermartingale, and an equality instead of the inequality in (24). This provides

$$
V(x, y)=\mathbf{P}(\tau=\infty),
$$

showing that $V(x, y)$ is indeed the value function of the problem, and $u_{t}^{*}$ the optimal strategy.

The two pictures in Figs. 1 and 2 show the gain of the collaboration for two parameter choices, i.e. the difference $V(x, y)-\left(1-e^{-2 \mu_{1} x}\right)\left(1-e^{-2 \mu_{2} x}\right)$, where the second term describes the probability that both companies survive, if there is no collaboration.

\section{Concluding remarks}

Although not direct comparable-since in these papers singular controls, modeling the accumulated transfer resp. dividend payments, are allowed, it is instructive to take a look at the structure of the optimal strategies in $[2,8]$.

In [2], where compound Poisson processes are used for the endowment, the strategy is rather complicated. Depending on the behavior of the value function the positive quadrant for the current endowments is divided into several subsets. On these subsets the following dividend strategies are optimal: no action at all, lump sum payment, payment at certain rates. Moreover, it is assumed that company one has always to cover the deficit of the other one, if company two is in trouble (and vice versa).

Fig. 1 Gain for the probability that both companies survive for the parameters $\delta=0.1$, $\mu_{1}=0.3, \mu_{2}=0.7$

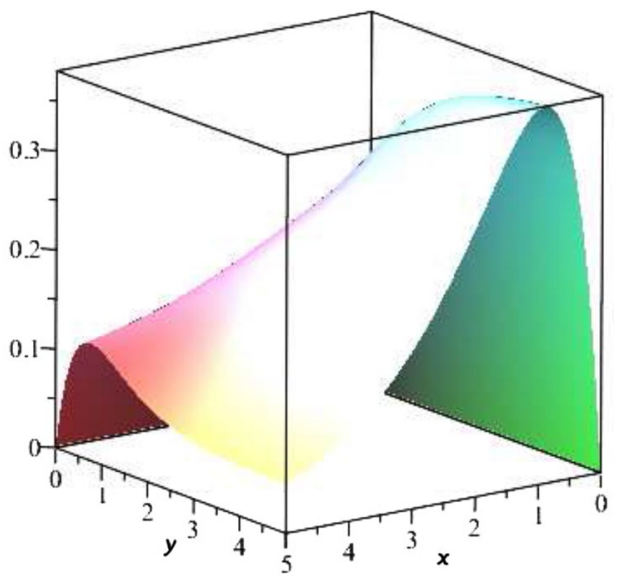


Fig. 2 Gain for the probability that both companies survive for the parameters $\delta=0.1$, $\mu_{1}=0.5, \mu_{2}=0.5$

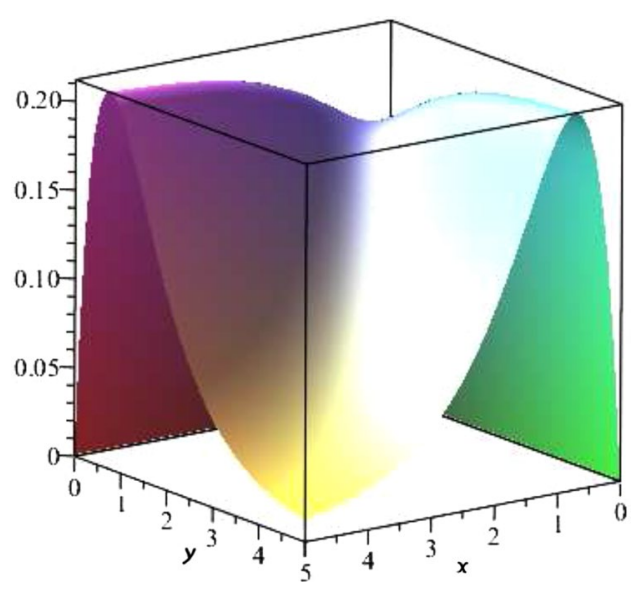

This "bailing out" assumption is also used in [8], where a diffusion approximation is used. Here the optimal strategy is easier: one possibility (the optimal strategy is not unique here) is, to keep the surplus of company 2 at zero at any time by continuously transferring money. The dividend payments are done by company one, using a barrier strategy. This solution corresponds to merging the two lines and solving a one-dimensional problem.

Let us finally remark that, allowing singular controls in our setting, ruin would occur only, if both companies are ruined simultaneously (similarly as in [8]), and the problem is again reduced to a one dimensional one.

Acknowledgements Open access funding provided by Austrian Science Fund (FWF). Support by the "Austrian Science Fund" (Fonds zur Förderung der wissenschaftlichen Forschung), Project nr. P30864-N35, is gratefully acknowledged. Moreover, I thank an anonymous referee for carefully reading the paper and suggestions to improve it.

OpenAccess This article is distributed under the terms of the Creative Commons Attribution 4.0 International License (http://creativecommons.org/licenses/by/4.0/), which permits unrestricted use, distribution, and reproduction in any medium, provided you give appropriate credit to the original author(s) and the source, provide a link to the Creative Commons license, and indicate if changes were made.

\section{References}

1. Asmussen S, Albrecher H (2010) Ruin probabilities, vol 14, 2nd edn. Advanced series on statistical science and applied probability. World Scientific Publishing, Hackensack

2. Albrecher H, Azcue P, Muler N (2017) Optimal dividend strategies for two collaborating insurance companies. Adv Appl Probab 49(2):515-548

3. Avanzi B (2009) Strategies for dividend distribution: a review. N Am Actuar J 13(2):217-251

4. Avram F, Palmowski Z, Pistorius M (2008) Exit problem of a two-dimensional risk process from the quadrant: exact and asymptotic results. Ann Appl Probab 18(6):2421-2449

5. Collamore J (1996) Hitting probabilities and large deviations. Ann Probab 24(4):2065-2078

6. Gerber HU, Shiu E (2006) On the merger of two companies. N Am Actuar J 10(3):60-67

7. Grandits $\mathrm{P}$ (2019) A two dimensional dividend problem for collaborating companies and an optimal stopping problem. Scand Actuar J 2019(1):80-96 
8. Gu J, Steffensen M, Zheng H (2018) Optimal dividend strategies of two collaborating businesses in the diffusion approximation model. Math Oper Res 43(2):377-398

9. McKean HP, Shepp LA (2006) The advantage of capitalism vs. socialism depends on the criterion. J Math Sci 139(3):6589-6594

Publisher's Note Springer Nature remains neutral with regard to jurisdictional claims in published maps and institutional affiliations. 